\title{
Das Nervensystem und die «sogenannte Seelentätigkeit» in der Lehre Pawlows*
}

Von Huldrych M. Koelbing

Ein entschiedenes Nein, ein kategorisches Ja

Das Bestreben, das Leben der Seele und seine Störungen von der somatischen Grundlage her zu erklären, dem wir in der hippokratischen Schrift «Von der heiligen Krankheit» zum erstenmal begegnen, setzt sich in der Wissenschaft immer wieder durch - ungeachtet der Tatsache, daß doch ein jeder von uns das eigene Erleben, Fühlen, Denken und Schaffen als etwas ganz Ursprüngliches empfindet und daß sich diese psychischen Vorgänge mit irgendwelchen materiellen Prozessen in unserem Nervensystem niemals wirklich identifizieren lassen. Die Erforschung des Seelenlebens als Hirnfunktion stellt eine Vereinfachung dar, die methodisch vollkommen gerechtfertigt ist, die aber die seelische Wirklichkeit nur von einer Seite her erfaßt. Der Forscher, der sich darin engagiert, wird allerdings von seinem Forschungsgegenstand so fasziniert, daß er sehr leicht alles übrige vergessen kann und den Teilaspekt, der seinen Methoden zugänglich ist, wenn nicht für das Ganze, so doch für das allein Wesentliche nimmt. Das wurde im bisherigen Verlauf des 20. Jahrhunderts vielleicht nirgendwo deutlicher als bei Iwan Petrowitsch Pawlow (1849-1936), der für seine Arbeiten zur Verdauungsphysiologie 1904 den Nobelpreis erhielt, in den folgenden Jahrzehnten aber immer ausschließlicher sich darum bemühte, die «sogenannte Seelentätigkeit» als ein System von Reflexen zu erfassen und auch ihre Störungen, die Neurosen und Psychosen, auf dieser Grundlage zu erklären.

Man könnte nun annehmen, Pawlow wäre beim Experimentieren mit den von ihm beim Hund entdeckten bedingten Reflexen ganz allmählich in diese Bahn

* Meinem verehrten Vorgänger, Lehrer und Freund Erwin H. Ackerknecht, der mich seinerzeit auf Pawlows psychiatrische Theorien aufmerksam gemacht hat, zum 70. Geburtstag gewidmet. (Vgl. E.H. Ackerknecht, Kurze Geschichte der Psychiatrie, Stuttgart 1957, Kap. 10.) 
gedrängt worden und die wachsende Fülle der Beobachtungen hätte ihn veranlaßt, unwillkürlich einen immer größeren Anteil der Nerventätigkeit (im weitesten Sinne) als Aktionsbereich der Reflexe zu sehen. Bei der Lektüre seiner Arbeiten zeigt es sich jedoch, daß es nicht die Versuchsergebnisse waren, die Pawlow nach und nach dazu führten, das Seelenleben auf somatische Mechanismen (eben die Reflexe) zurückzuführen, sondern daß er von allem Anfang an dazu entschlossen war, in dieser Richtung vorzugehen.

Pawlow hat die verschiedenen Veröffentlichungen über seine Reflexlehre und ihre Anwendung auf Psychologie und Psychiatrie 1923 in den «Zwanzigjährigen Erfahrungen mit dem objektiven Studium der höheren Nerventätigkeit (des Verhaltens) der Tiere» zusammengefaßt und dieses Buch von der 2. bis zur 6. Auflage (diese erschien postum 1938) durch neue Aufsätze und Reden schließlich auf das Doppelte des ursprünglichen Umfangs erweitert ${ }^{1}$. Doch schon der erste Beitrag, der in die «Zwanzigjährigen Erfahrungen» aufgenommen wurde ein Vortrag aus dem Jahr 1903, gehalten vor dem Internationalen Medizinischen Kongreß in Madrid - trägt den programmatischen Titel «Experimentelle Psychologie und Psychopathologie bei Tieren $»{ }^{2}$ Dabei wird die Untersuchung der Speichelsekretion bei «systematischer Zergliederung und Zerstörung des Zentralnervensystems» als eine höchst aussichtsreiche Methode der «experimentellen Psychopathologie» empfohlen; vor allem aber macht Pawlow in diesem Vortrag seinen mechanistischen Standpunkt klar. Die grundsätzliche Frage stellte sich schon, als Pawlows Mitarbeiter S.G.Wulfson nachwies, daß schon das bloße Vorzeigen von bestimmten Dingen genügt, um bei Hunden eine Speichelsekretion auszulösen.

«Ein Hund sieht, spürt, riecht diese Stoffe, wird auf sie aufmerksam, er strebt zu ihnen, wenn es genießbare und angenehme Stoffe sind, er wendet sich von ihnen ab, widersetzt sich ihrer Einführung, wenn es unangenehme Stoffe sind. Jeder wird sagen, daß dies eine psychische Reaktion von Seiten des Tieres ist, daß es sich um eine psychische Anregung der Speicheldrüsentätigkeit handelt.»

Was aber soll nun der Physiologe mit diesen Beobachtungen anfangen? «Müssen wir uns zum Verständnis dieser neuen Erscheinungen in den inneren Zustand des Tieres versetzen, uns seine Empfindungen, Gefühle und Wünsche analog den unseren vorstellen? - Für den Naturwissenschaftler bleibt, wie mir scheint, auf diese letzte Frage nur eine Antwort, ein entschiedenes 'Nein'.» Für ihn stellt sich die Frage nach dem Verständnis der psychisch bedingten Reaktionen in folgender Form: «Kann man dieses ganze scheinbare Chaos von Beziehungen in einen bestimmten Rahmen bringen, die Erscheinungen reproduzierbar machen, ihre 
Regeln und ihren Mechanismus aufdecken?" Pawlow glaubt sich schon jetzt aufgrund seiner bisherigen Versuche berechtigt, «auf diese Frage mit einem kategorischen ' $J a^{\prime}$ ' zu antworten und die Feststellung zu treffen, daß allen psychischen Versuchen immer derselbe spezielle Reflex als wesentlicher und allgemeiner Mechanismus zugrunde liegt.»

Ganz kampflos ist Pawlow freilich nicht zu diesem Standpunkt gelangt. In der Einleitung zu seinem Buch äußert er sich darüber ${ }^{3}$. Während Wulfson nach den ersten Versuchen neues Material über die «psychische Erregung der Speicheldrüsen» sammelte, versuchte Pawlows zweiter Mitarbeiter, A.T.Snarski, den inneren Mechanismus dieser Erregung zu analysieren.

«Dabei vertrat er einen subjektiven Standpunkt, d.h. er berücksichtigte die imaginäre innere Welt der Hunde (...) mit ihren Gedanken, Gefühlen und Wünschen analog unserer Innenwelt. Da ereignete sich ein im Laboratorium nie dagewesener Fall. Wir gingen in unserer Deutung dieser Welt scharf auseinander und konnten uns durch keinerlei weitere Versuche auf irgendeine gemeinsame Schlußfolgerung einigen, ungeachtet der ständigen Laborpraxis, in der neue Versuche, die mit beiderseitigem Einverständnis unternommen werden, gewöhnlich jegliche Meinungsverschiedenheiten und Streitigkeiten entscheiden. Dr. Snarski blieb bei der subjektiven Deutung der Erscheinungen. Ich aber, betroffen durch das Phantastische und die wissenschaftliche Nutzlosigkeit einer solchen Einstellung zur gestellten Aufgabe, begann einen anderen Ausweg aus der schwierigen Lage zu suchen. Nach beharrlichem Nachdenken über das Problem, nach einem schweren geistigen Kampf habe ich endlich beschlossen, auch gegenüber der sogenannten psychischen Erregung in der Rolle des reinen Physiologen zu bleiben, d.h. in der Rolle des objektiven äußeren Beobachters und Experimentators, der es ausschließlich mit äußeren Erscheinungen und ihren Beziehungen zu tun hat.»

Damit ist ganz eindeutig festgestellt - und zwar durch einen sehr bedeutenden Experimentator -, daß sich auch in der experimentellen Forschung die Interpretation eines biologischen Versuches nicht ohne weiteres aus dem Resultat von selbst ergibt, sondern daß sie oft eine Vorentscheidung verlangt, nämlich den Entschluß, die Dinge auf die eine oder die andere Art zu betrachten. Pawlow hat diese Vorentscheidung ganz bewußt getroffen; daß aber schon damit unvermeidlicherweise ein persönliches, subjektives Moment, nämlich seine eigene Wissenschaftsphilosophie, in seine Forschungsarbeit hereinkam, hat er sich nicht eingestanden. 


\section{Die Persönlichkeit und ihre Erkrankungen in Pawlows Reflexpsychologie}

Pawlows Entschluß, sich bei der Erforschung psychischer Phänomene an die objektiv erfaßbaren, experimentell reproduzierbaren Äußerungen der Lebewesen zu halten - er fühlte sich darin in Übereinstimmung mit dem amerikanischen Psychologen Edward Lee Thorndike (1874-1949) ${ }^{4}$ - , war methodisch durchaus korrekt. Damit war aber auch die Möglichkeit zuverlässiger Aussagen auf die Äußerlichkeiten des Verhaltens und die denselben zugrunde liegenden neurophysiologischen Mechanismen beschränkt. An eine solche Beschränkung glaubte Pawlow indessen nicht. Von einer "psychischen Tätigkeit der höheren Tiere» darf der Naturforscher seiner Meinung nach zwar nicht reden ${ }^{5}$, aber ${ }^{6}$ : «Die erhaltenen objektiven Angaben wird die Wissenschaft, von der Ähnlichkeit oder Identität der äußeren Erscheinungen geleitet, über kurz oder lang auch auf unsere subjektive Welt übertragen. Damit wird sie plötzlich unsere so geheimnisvolle Natur klar beleuchten und den Mechanismus und Lebenssinn dessen klären, was den Menschen am meisten beschäftigt, sein Bewußtsein, die Konflikte seines Bewußtseins.» Der physiologische Begriff des Reflexes wurde für Pawlow zum Schlüssel, der eben doch die Geheimnisse des Seelenlebens dem menschlichen Verständnis erschließen sollte. Was dabei herauskam, soll nun in zwei Beispielen skizziert werden.

\section{a) Struktur der Persönlichkeit}

Grundlage des Verhaltens - als der faßbaren Äußerung der «sogenannten Seelentätigkeit» - sind die angeborenen, unveränderlichen, unbedingten Reflexe (die man sonst als Instinkte bezeichnete). Im Verlauf des Lebens bildet sich auf dieser Basis ein Überbau von Gewohnheiten und Assoziationen; sie sind in Wirklichkeit ebenfalls Reflexe, aber erworbene, veränderliche, bedingte Reflexe ${ }^{7}$.

Unter den unbedingten Reflexen anerkennt Pawlow auch einen «Freiheitsreflex $» .^{8}$ Er äußert sich im anfänglichen Widerstand mancher Versuchstiere gegen die Einengung ihrer Bewegungsfreiheit beim Experimentieren und ist für das Individuum sehr wichtig, denn sonst würde es sich in seinem Tun von jedem Widerstand sofort völlig hemmen lassen. Das Gegenstück zu diesem «Freiheitsreflex » stellt der «Servilitätsreflex» dar, der sich in der sklavischen Unterwerfung eines schwachen Tieres unter die Gewalt des stärkeren äußert und der selbstverständlich auch in der menschlichen Gesellschaft seine Rolle spielt: 
«Wie oft und verschiedenartig tritt der Servilitätsreflex auf russischem Boden in Erscheinung, und wie nützlich ist es, sich dessen bewußt zu werden! Führen wir ein Beispiel aus der Literatur an. In der kleinen Erzählung Kuprins 'Der Strom des Lebens' wird der Selbstmord eines Studenten beschrieben, der von seinem Gewissen gepeinigt wurde, weil er seine Kameraden an die Geheime Staatspolizei verraten hatte. Aus dem Brief des Selbstmörders geht klar hervor, daß der Student ein Opfer des Servilitätsreflexes wurde, den er von seiner Mutter, einer Gnadenbrotempfängerin, geerbt hatte. Hätte er das gut verstanden, so hätte er erstens gerechter über sich geurteilt, und zweitens hätte er durch systematische Maßnahmen eine erfolgreiche Hemmung, eine Unterdrückung dieses Reflexes entwickeln können.»

Der Pawlowschen Reflexpsychologie - das zitierte Beispiel macht es deutlich wohnt wie jeder deterministischen Psychologie die Tendenz inne, die Entscheidungsfreiheit des Menschen und damit seine Verantwortung für sein Tun zu negieren. Eine Persönlichkeit, die aus einigen unbedingten und sehr vielen bedingten Reflexen besteht, muß zudem in höchstem Grade durch die Umweltbedingungen formbar sein. Diese Konsequenz der Pawlowschen Theorie fügte sich sehr gut in die marxistische Auffassung, die den Menschen als das Produkt seiner sozialen Umwelt sieht. Infolgedessen erhielten Pawlows Lehren (obwohl er selbst dem bolschewistischen Regime gegenüber lange kritisch eingestellt blieb $^{9}$ ) in der Sowjetunion den Rang einer wissenschaftlichen Orthodoxie und waren damit trotz ihres spekulativen Charakters der freien wissenschaftlichen Diskussion entzogen.

\section{b) Temperamente, Neurosen und Psychosen ${ }^{10}$}

Im Verlauf seiner Experimente wurde es Pawlow klar, daß die Hemmungsprozesse für die Funktion des Nervensystems ebenso wichtig sind wie die Erregungsprozesse; das «ständige richtige Ausbalancieren dieser beiden Prozesse» macht das normale Leben des Menschen wie der Tiere aus ${ }^{11}$. Rief man nun im Experiment einen «schweren Zusammenstoß des Erregungs- und Hemmungsprozesses» hervor, indem man entgegengesetzt wirkende Reize einander unmittelbar folgen ließ, so reagierten die Hunde verschieden. Manche wurden krank, und zwar gerieten einige in eine Dauererregung, die Pawlow der menschlichen «Neurasthenie» gleichsetzte, während sich bei den anderen ein anhaltender Zustand der Hemmung einstellte, den er als «Hysterie» diagnostizierte. Zwischen diesen gegensätzlichen Typen hielt sich eine Gruppe von Hunden, die auch bei dieser Überbeanspruchung ihres Nervensystems gesund und ausgeglichen blieben, und hier 
ließen sich wiederum zwei Naturen unterscheiden: die lebhaften und die ruhigen. Also vier in ihrer Konstitution unterschiedliche Typen, die offenbar dem entsprachen, was man beim Menschen seit Hippokrates' Zeiten als Temperamente bezeichnete!

Temperamente und ihre Psychopathologie nach Pawlow

\begin{tabular}{|c|c|c|c|c|}
\hline \multirow{2}{*}{$\begin{array}{l}\text { Verhalten der } \\
\text { Hunde im } \\
\text { Experiment }\end{array}$} & \multirow{2}{*}{$\begin{array}{l}\text { übermäßig } \\
\text { erregbar }\end{array}$} & \multicolumn{2}{|c|}{ ausgeglichen - } & \multirow{2}{*}{$\begin{array}{l}\text { übermäßig } \\
\text { hemmbar }\end{array}$} \\
\hline & & - lebhaft & - ruhig & \\
\hline $\begin{array}{l}\text { Temperamente } \\
\text { bei Hund und } \\
\text { Mensch }\end{array}$ & cholerisch & sanguinisch & phlegmatisch & melancholisch \\
\hline $\begin{array}{l}\text { Neurosen } \\
\text { bei Hund und } \\
\text { Mensch }\end{array}$ & Neurasthenie & & & Hysterie \\
\hline $\begin{array}{l}\text { Psychosen } \\
\text { des Menschen }\end{array}$ & $\begin{array}{l}\text { zirkuläres } \\
\text { Irresein }\end{array}$ & & & Schizophrenie \\
\hline
\end{tabular}

Pawlow übernahm diesen Begriff aus der antiken Säftelehre ohne Bedenken: das Temperament, nun verstanden als die besondere Reaktionsbereitschaft des Nervensystems, erschien ihm als dessen "grundlegendste Charakterisierung»; das Temperament «gibt der gesamten Tätigkeit jedes Individuums ein ganz bestimmtes Gepräge».

Ohne Bedenken setzte Pawlow auch die menschlichen Neurosen seinen experimentellen Hunde-Neurosen gleich: Nach dem methodischen Prinzip, komplizierte Erscheinungen auf einfachere, elementarere zurückzuführen, «müssen auch die menschlichen Neurosen mit Hilfe der Tierneurosen als den naturgemäß einfacheren gedeutet und verstanden, d.h. analysiert werden ... " $^{12}$

Daraus ergab sich: Überfordert man das Nervensystem eines Menschen wie dasjenige eines Versuchshundes, dann reagiert der erregbare Typ, der Choleriker, mit Neurasthenie, der hemmbare Typ dagegen, der Melancholiker (dessen Hirnrinde zu schwache Nervenzellen besitzt), mit Hysterie. Die ausgeglichenen Typen, 
der lebhafte Sanguiniker und der ruhige Phlegmatiker, lassen sich nicht aus dem Gleichgewicht bringen und werden infolgedessen auch nicht - oder doch nicht so leicht - neurotisch. Man bedenke: es war ein Zeitgenosse Sigmund Freuds, der diese neurophysiologische Neurosetheorie entwickelte!

Nachdem er den Schritt in die menschliche Psychopathologie einmal getan hatte, ging Pawlow noch etwas weiter und bezog auch die beiden Hauptformen der endogenen Psychosen in sein Schema ein ${ }^{13}$. Das zirkuläre Irresein wird in dieser Sicht zur übersteigerten «Neurasthenie», die Schizophrenie zum höchsten Grad der «Hysterie» (und damit paradoxerweise auch zur Psychose des melancholischen Temperamentes!).

Beachtlicher als diese Spekulationen sind Pawlows Überlegungen über die Fragen, die sich Ärzte und Forscher angesichts der neurotischen Patienten stellen müssen: Nachdem man zuerst einmal genau bestimmt hat, worin denn im gegebenen Fall die Abweichung von der Norm mit ihrer großen Schwankungsbreite liegt, muß man gemeinsam mit dem Patienten oder auch gegen seinen Widerstand im Gewirr der Lebensbeziehungen nach denjenigen Bedingungen und Umständen suchen, die vernünftigerweise mit dem Ursprung der Neurose in Verbindung gebracht werden können. Darauf ist zu klären, warum diese Bedingungen und Umstände nun gerade bei diesem Kranken pathogen gewirkt haben, während sie bei anderen Menschen in vergleichbarer Lage ohne Wirkung blieben oder aber zu einem anderen Zustandsbild führten.

Doch damit ist das Problem erst halb gelöst. Die pathogenen Lebensumstände und -eindrücke wirken ja auf das Nervensystem. Im Sinne der Pawlowschen Lehre muß man daher fragen ${ }^{14}$ : «Welche Lebensbedingungen waren übermäßig stark für das gegebene Nervensystem, wann und wo stießen die Anforderungen an seine Tätigkeit und an seine Hemmungsfunktion in unerträglicher Weise zusammen?» Das ist für Pawlow der entscheidende Punkt in der Entstehung einer Neurose; «die unzähligen Erlebnisse des Neurotikers» stellen dagegen nur «Variationen ein und desselben physiologischen Prozesses» dar und sind als solche belanglos. Neurose oder Psychose entsteht also aus der Einwirkung sozialer Belastungsfaktoren auf ein Nervensystem von bestimmter Konstitution.

Pawlows Lehre verkennt die endogene, schicksalshafte Natur der großen Psychosen und mißachtet die persönlichen Momente der individuellen Lebensgeschichte für die Entstehung der Neurosen und psychosomatischen Störungen. Aber an der Frage, die Pawlow zu lösen versuchte, nämlich wie denn psychische Einflüsse in die Bahnen des somatischen Geschehens geleitet werden, darf die psychosomatische Medizin nicht vorübergehen, wenn sie wissenschaftlich bleiben will. 


\section{Anmerkungen}

1 Pawlow, I.P., Sämtliche Werke, Bde. III/1 und III/2. Berlin (AkademieVerlag) 1953.

2 Op. cit., III/1, S. 9-21.

Op.cit., III/1, S. If. (Hervorhebung im Text von mir).

Op. cit., III/1, S. 2 f.

Op. cit., III/1, S. 42.

Op. cit., III/1, S. 21.

7 In der Einleitung (III/1, S. 2) nennt Pawlow als entscheidenden Anstoß für sein Forschen und Denken Iwan Michailowitsch Setschenows «Reflexe des Gehirns» von 1863. - In einem Aufsatz «Über psychische Reflexactionen» hatte der junge Psychiater und Internist Wilhelm Griesinger schon 1843 die Hirntätigkeit analog zur Reflextätigkeit des Rückenmarks zu erklären versucht.

$8 \quad$ Pawlow, I.P., Der Freiheitsreflex (1917, mit M.M.Gubergriz), in: Sämtliche Werke, III/1, S. 248-252. - Zitat von S. 252.

9 Vgl. Babkin, B.P., Pavlov, a biography, Chicago (University of Chicago Press) 1949, S.161ff.

10 Pawlow, I.P., Die physiologische Lehre von den Typen des Nervensystems, den Temperamenten (1927/28), in: Sämtliche Werke III/2, S. 345-353.

11 In diesem Zusammenhang wären - neben Setschenow - die Arbeiten C.S. Sherringtons zu nennen, der schon früher das richtige Zusammenspiel von Erregung und Hemmung als wesentliche Voraussetzung für die Integrationsfunktion des Zentralnervensystems erkannt hatte. Vgl. sein Buch: The integrative action of the nervous system, New Haven (Yale University Press) 1906.

12 Pawlow, I.P., Sämtliche Werke III/2, S. 399.

13 Pawlow, I.P., Die physiologische Lehre von den Typen ..., Op.cit., III/2, S. $352 \mathrm{f}$.

14 Pawlow, I.P., Über die Neurosen des Menschen und des Tieres (1932), in: Sämtliche Werke III/2, S. 399-401 - Zitat von S. 401.

\section{Summary}

An important point in Pavlov's work on the "so-called psychic life" of higher animals (including man) is his own statement that, in order to interpret his experimental observations, he deliberately followed a bias: after "a hard mental struggle", he decided not to admit the influence of such subjective factors as emotions on the animals' behaviour, but to describe the latter exclusively in objective physiological terms. As a methodological restriction, this decision was perfectly legitimate, but Pavlov could not resist the temptation to develop from 
this narrow line of approach an allegedly comprehensive psychology. Two examples of this are discussed, which show clearly the highly speculative nature of this supposedly objective thinking: (a) According to Pavlov, personality is just a system of reflexes-inborn and unconditioned as well as acquired and conditioned; personal responsability and guilt are reduced to the effects of the unadjusted "liberty" and "servility reflexes". (b) Neuroses and psychoses are seen as the results of the individual's inability to cope with contradictory external stimuli. In spite of the grotesque inadequacy of this explanation, the problem that Pavlov tried to solve is a very real one, and Western psychiatry and psychosomatic medicine should take it more serious than it sometimes does: which are, physiologically speaking, the channels by which emotions cause somatic disease?

Prof. Dr.med. Huldrych M. Koelbing

Medizinhistorisches Institut der Universität Zürich

Rämistraße 71

8006 Zürich 